\title{
DYNAMICALLY PRIORITIZED VIRTUAL-NETWORK MONITORING ACCORDING TO LIFECYCLE OF VIRTUAL MACHINES IN LARGE SCALE DATA CENTER
}

\author{
Hideki Okita ${ }^{1}$, Hayato Hoshihara ${ }^{2}$, Norihisa Komoda ${ }^{3}$ and Toru Fujiwara ${ }^{3}$ \\ ${ }^{1}$ Research and Development Group, Hitachi, Ltd., 1-280 Higashi-Koigakubo, Kokubunji, Tokyo, 185-8601, Japan \\ ${ }^{2}$ IoT \& Cloud Services Business Division, Hitachi, Ltd., 6-26-3 Minamioi, Shinagawa-ku,, Tokyo, 140-0013, Japan \\ ${ }^{3}$ Graduate School of Information Science and Technology, Osaka University, 1-5 Yamadaoka, Suita, Osaka, \\ 565-0871, Japan
}

\begin{abstract}
We propose a life cycle-based dynamic virtual network monitoring system that focuses on the varying failure rate in the life cycle of the VM and selects the target device for dynamic and continuous network monitoring. When using a combination of server monitoring with ping and network connection check with Ethernet OAM, the system sets higher priorities to the port connected to a long running server, and the port within a certain time after the connection change or virtual machine addition is set. The system then selects the ports from the higher priority port to be monitored by Ethernet OAM. As a result of the evaluation by the simulation, by dynamically selecting the port to be monitored for Ethernet OAM using the proposed method, it was confirmed that the average locating time of the VM failure is reduced by $2.2 \%$ compared to the conventional method. In the data center for cloud services running many VMs, it is possible to shorten the recovery from VM failure while suppressing the number of objects monitored by Ethernet OAM by using this method.
\end{abstract}

\section{KEYWORDS}

Virtual Network, Network Management, Data Center, Cloud Service, OAM

\section{INTRODUCTION}

As cloud services are applied to wider business fields, the demand for stable operations of IT systems in data centers that provide cloud services is growing. It requires not only higher reliability of the IT systems and but also rapid recovery to the normal state at the failures of the IT systems. The rapid recovery requires not only rapid detection of the failures and but also rapid locating the points of failure among various devices such as servers and network switches in data centers.

The load on administrators to manage the devices increases as the number of monitored devices in IT systems increases. The number of monitored devices is increasing especially in the data centers of cloud service providers and large companies due to the adoption of server virtualization technologies which enables to deploy multiple logical servers, called virtual machines (VMs), on a physical server. Also, network function virtualization (NFV) which virtualizes networks by virtual network function (VNF) is introduced in data centers. Network devices such as proxy servers, firewall devices, load balancers, and WAN optimization devices are running as VMs on servers (Luizelli, M.C. et al, 2015).

Communication protocols are standardized and widely used to monitor remotely server availability and network connectivity in data centers. In particular, ICMP (Internet Control Message Protocol) is the standard protocol for network management systems to monitor servers and network devices in TCP/IP networks by exchanging monitoring packets, well known as 'ping' (Postel, J., 1981).

Also, Ethernet OAM (Operations, Administration and Maintenance) is developed to monitor connectivity in Ethernet-based wide-area networks (WANs) (McFarland, M. et al, 2005). IEEE802.1ag (IEEE 802.1 WG, 2007) and ITU-T Y.1731 (ITU-T, 2015) are defined as the standard of Ethernet OAM. Ethernet OAM functions are generally implemented in network devices. Ethernet OAM supported devices transmit and receive continuously monitoring frames each other to check connectivity. 
However, as monitored devices increase, more resources are necessary for monitoring systems although the resources of the monitoring system available for these monitoring processes are limited. Therefore, the data centers of cloud service providers and large enterprises require a network-based monitoring method that provides short average failure locating time for many VMs with less resources.

In this paper, we focus on the varying VM failure rates for each stage of the lifecycle of the monitored VMs. We propose a method to model the varying failure rate of the VM and to elect the target to focus on based on the failure rate. The following sections of this paper are constructed as follows. Section 2 describes related research works to monitor virtual networks. Section 3 describes an efficient virtual network monitoring system based on the life cycle of physical servers and VMs in the data center. Section 4 describes the evaluation results of the effectiveness of the proposed method by simulation. In addition, we examine the characteristics of the assumed model parameters when they are different from the model parameters being monitored.

\section{RELATED WORK}

The network problem is a major factor of service failures and accounted for $76 \%$ of the failure factors according to the failure analysis in a large-scale Internet service (Oppenheimer, D. et al, 2003). Also, the failures due to network problems are not insignificant according to the investigation results of the research institution (Schroeder, B. and Gibson, G.A., 2006) and the survey result in a commercial data center (Birke, R. et al, 2014).

Anomaly detection methods for network devices based on passive measurement realize precise and sensitive failure detection by using various statistical information (Tang, Y. et al, 2005; Gomes, R.L. et al, 2016; Katzela, I. and Schwartz, M., 2015; Liu, D. et al, 2013; Thottan, M. and Ji, C., 2003; Lakhina, A. et al, 2005; Mi, H. et al, 2013), although those require more monitoring resources of the network monitoring systems. From the active measurement viewpoint, a framework for achieving proactive network management is developed to predict exceptions in IP/MPLS networks by using OAM functions (Dini, P. et al, 2004). Also, a framework to integrate service-level monitoring with fault management using Ethernet OAM is developed for interconnected networks of Ethernet service providers so that they can identify rapidly the root cause in the networks (Varga, P. and Moldovan, I., 2007). However, these studies focus on networks with fixed monitoring targets for network service providers.

Also, the number of network end points that a network switch can monitor with Ethernet OAM at once is limited to at most tens due to the limitation of hardware resource such as a CPU of the network switch. As the number of monitored end points increases, the network switch receives more Continuous Check (CC) messages from the end points. It causes increasing CPU usage of the network switch to process the messages. For example, the maximum number of the monitored end points that a network-switch product can monitor in a network is set to 100 in default.

Therefore, the data center of a large-scale cloud service provider that creates and runs thousands of VMs and hundreds of thousands of VLANs, can not set all VMs and VLANs to be monitored with Ethernet OAM at the same time. As a result, while ping is used for all servers, the Ethernet OAM is used for limited servers. Therefore, servers that are not monitored with Ethernet OAM must be performed the connection check of the corresponding network part again once the failure of the server is detected at the service level by ping. There is a problem that it takes time to isolate the failure cause between the server and the network.

\section{DINAMICALLY PRIORITIZED VIRTUAL NETWORK MONITORING}

\subsection{Method Overview}

In this paper, we propose a new method of monitoring to reduce the average server failure locating time. Figure 1 shows the failure management in the method in a data center with Ethernet OAM and ping. Usually, there are two types of administrators in a data center; server administrators who use ping to monitor servers and network administrators who use Ethernet OAM to monitor networks in the data center. They should cooperate to clarify the root cause of service failures in the data center. When a server administrator detects a service failure by missing responses of ping messages from a monitoring server, the root cause of the failure is not yet 
clarified. A network administrator thus explores the Ethernet OAM monitoring point (MEP: Maintenance End Point) of the port that the server is connected. If the network administrator finds a failure with Ethernet OAM, the cause of the service failure exists in the network side. On the other hand, if Ethernet OAM does not detect a failure, it is considered that the cause of the service failure exists in the server side.

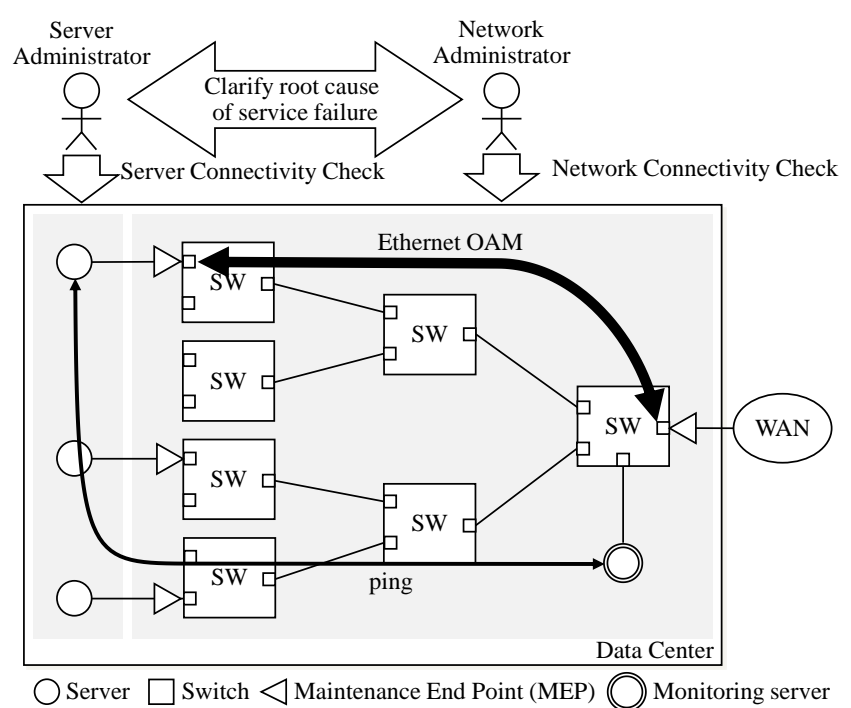

Figure 1. Failure Management of Data Center with Ping and Ethernet OAM

We introduce a new concept of dynamically selecting VMs to be monitored based on the stage of the VM lifecycle. In the past, the monitoring priorities of VMs are fixed throughout the lifecycle. However, when an IT system deploys a new physical server to activate a VM and changes its settings, if the elapsed time since it was activated is long, the hardware running the VM is likely to fail. Therefore, by increasing the allocation monitoring resources for VMs in such stage, and by decreasing the allocation monitoring resources for VMs in other stages, it is possible to shorten the average failure detection without increasing monitoring resources.

In order to select the monitored port, it is necessary to define and calculate the priority which changes in the time series according to the stage of the life cycle for each VM as an indicator of the selection. Therefore, the monitoring priority of the VM is changed according to the time series change of failure rate per VM. The pattern of failure rate for the operating time of a typical hardware including HDD on the server in the data center follows the so-called bathtub curve. The failure rate showed a high value early, decreased and eventually stabilized to a low value over time, and the high value is again shown by wear at the end of the life cycle (Yang, J. and Sun, F.B., 1999; Schroeder, B. and Gibson, G.A., 2007). Therefore, since the initial failure rate is high at the time of the introduction of the server, the monitoring priority of the VM running on the server is high, and the priority due to the initial failure is lowered over the period. On the other hand, the monitoring priority rises due to hardware wear over time.

Figure 2 shows which VMs in the data center are selected for monitoring based on the monitoring priority calculated for each VM, and also shows the time variation of the selected VM combinations. The four graphs of the left part of Figure 2 show the time variation of the monitoring priority for each of the four VMs. In addition, the thick lines of each graph represent the state in which the VM was selected for monitoring. 


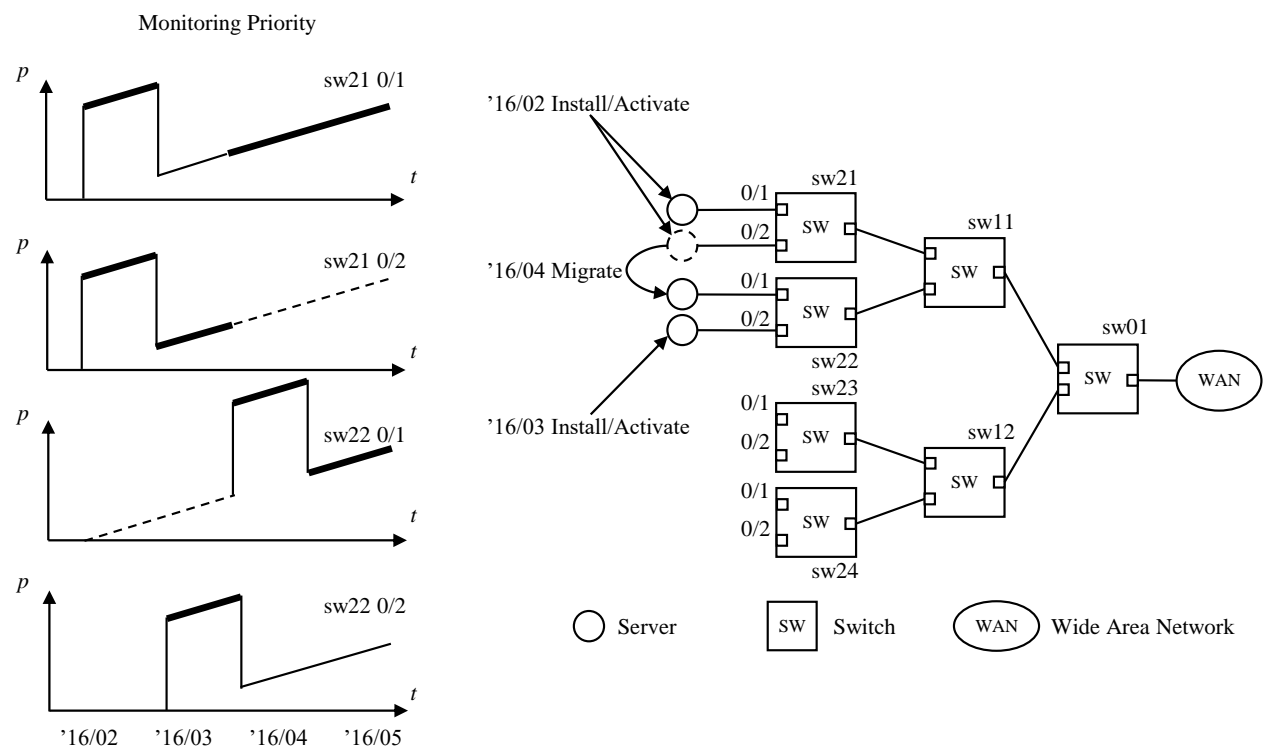

Figure 2. VM Monitoring Priority

In this example, the number of MEPs that can be allocated is limited to two. Two MEPs are first allocated to the network switch SW21 to monitor the connectivity to the ports connected to two servers when the servers activated two VMs at Feb. '16. When the third server with a VM is connected to the network switch SW22 at Mar. '16, the port that connects the first server is excluded from the monitored ports. And the port that connects the third server is added to the monitored ports. In addition, when the VM on the second server moves to the third server at Apr. '16, the priority of the port of the network switch SW22 that the third server is connected is increased to reflect the rise of the failure rate due to the configuration change, and the port is added to the monitored ports. At the same time, the port of the network switch SW21 connecting the first server is added again to monitored ports as the failure rate of the first server rises.

\subsection{Port Monitoring Priority}

To prioritize the above mentioned monitored ports, the proposed method calculates the port monitoring priority for each port of the switch in the network based on the total failure rate. The port monitoring priority $p$ can be expressed as the following equation using the server wear failure rate $\lambda_{\text {wear }}(\mathrm{t})$, server initial failure rate $\lambda_{\text {init }}(\mathrm{t})$, and server change-derived failure rate $\lambda_{\text {chg }}(\mathrm{t})$.

$$
p=1-\left(1-\lambda_{\text {wear }}(t)\right)\left(1-\lambda_{\text {init }}(t)\right)\left(1-\lambda_{\text {chg }}(t)\right)
$$

The method calculates the failure rate of the server wear-out failure $\lambda_{\text {wear }}(t)$ as following. Since the components of the server wears out as time goes on, the failure rate increases as well. The parameters $a$ and $t_{1}$ represent the speed of wear-out and the time when the server has been deployed, respectively.

$$
\lambda_{\text {wear }}(t)=\left\{\begin{array}{cc}
1-e^{-a\left(t-t_{1}\right)} & \left(t \geq t_{1}\right) \\
0 & \left(t<t_{1}\right)
\end{array}\right.
$$

The method then calculates the server initial failure rate $\lambda_{\text {init }}(\mathrm{t})$ from the uptime of the server. The failure rate $\lambda_{\text {init }}(\mathrm{t})$ of a server is assumed to follow the following equation. Since the initial failure decreases as time goes on, the failure rate decreases as well. The parameter $\sigma_{\text {init }}$ indicates the speed of stability of the server. By such an expression, it expresses the state that the service failure occurs due to a configuration error for a certain time since the server has been deployed or changed or a virtual machine is deployed on the server.

$$
\lambda_{\text {init }}(t)=\frac{W_{\text {init }}}{\sqrt{2 \pi}} e^{-\frac{\left(t-t_{1}\right)^{2}}{2 \sigma_{\text {init }}{ }^{2}}}
$$

Then, this method checks whether the selection port is moved from the server connection change information, and that the date and time are entries prior to the specified times. If there is an appropriate entry, we calculate the failure rate $\lambda_{\text {chg }}(t)$ from the server change based on the elapsed time since the server connection 
occurred for each entry. If there is no entry, this method proceeds without calculating the failure rate $\lambda_{\text {chg }}(t)$ of the server changes. The failure rate $\lambda_{\mathrm{chg}}(\mathrm{t})$ is expressed as a failure rate that combines the failure rates caused by individual connection changes as following. The failure rate caused by each connection change is represented by a normal distribution centered on $t=t_{m}(m=0,1,2, \ldots)$ which is the timing of $m^{\text {th }}$ connection change.

$$
\lambda_{\text {chg }}(t)=W_{\text {chg }}-W_{\text {chg }} \prod_{m}\left\{1-\frac{1}{\sqrt{2 \pi}} e^{-\frac{\left(t-t_{m}\right)^{2}}{2 \sigma_{\mathrm{chg}}}}\right\}(m=0,1,2, \cdots)
$$

\subsection{MEP-enabled Port Selection}

The network monitoring system using the proposed method chooses which port of the network switch of the data center is monitored for the Ethernet OAM based on the abovementioned port monitoring priority. The network monitoring system, based on the port monitoring priority calculated in the manner described above, selects the port to be monitored of all ports connected to the server. In this case, the system selects the monitored port so that it does not exceed the maximum programmable MEP number of each switch.

\section{EVALUATION OF MONITORING EFFICIENCY}

\subsection{Evaluation Method}

By simulation, we compared the average failure locating time of each server in the following three cases; (1) dynamically changed monitoring by Ethernet OAM based on the proposed method, (2) fixed monitoring the server running an important application, and (3) ping without Ethernet OAM. For this simulation, a small-scale discrete event network simulator was developed in $\mathrm{C}++$. When this simulator is started, the object of the network node with the start-up time information is generated for each server. For each time step, the simulator checks if server failure happened or not for each object based on its uptime, configuration changes and the probability distribution of server failure. Also, it selects dynamically the objects that are monitored by using Ethernet OAM with MEP from all objects according to the proposed method in case (3). At the same time, the simulator checks if the server failures are detected with Ethernet OAM. If MEP is configured for a network node in failure, the Ethernet OAM detects quickly the failure. On the other hand, if MEP is not configured, the failure is detected by ping as usual. Based on the results, the simulator calculates the average failure locating time for the entire data center. The parameters of the implemented simulation are shown in Table 1.

Table 1. Simulation parameters

\begin{tabular}{ll}
\hline Parameter & Value \\
\hline Number of nodes & 1,000 \\
Simulation duration & 5 years \\
Simulation step interval & 7 days \\
Server connection change frequency & Once in 100 days \\
Ethernet OAM CC interval & 1 minute \\
Ping interval & 15 minutes \\
\hline Wear-out failure gradient $a$ & $1.0 \mathrm{e}-9$ \\
Initial failure rate amplitude $W_{\text {init }}$ & 0.0125 \\
Initial failure rate standard deviation $\sigma_{\text {init }}$ & $8.0 \mathrm{e} 4$ \\
Change failure rate amplitude $W_{\text {chg }}$ & 0.05 \\
Change failure rate standard deviation $\sigma_{\text {chg }}$ & $8.0 \mathrm{e} 4$ \\
\hline
\end{tabular}




\subsection{Evaluation Results}

We evaluate the improvement of the server failure locating time and the server failure detection rate by the proposed method. The server failure locating time is composed of the time that the monitoring system took for detecting a server failure by ping and the time that a network administrator took for identifying the network-side or the server-side as the cause of the server failure by using Continuity Check (CC) or Loop Back (LB) functions of Ethernet OAM. The server failure detection rate is defined as the percentage of servers that have been set to be monitored by using $\mathrm{CC}$ of Ethernet OAM with MEP among the servers that failed at the data center during the simulation.

Table 2 shows the simulation results of the server failure locating time and the server failure detection rate by the monitoring system. It shows, per server replacement rate, the number of failures, the server failure detection rate in the conventional method, and the server failure detection rate in the proposed method. As the server replacement rate increases, the number of total failures is reduced from 1357.65 to an average of 213.47. The average running time of the server is shortened as the server replacement rate increases. The server failure decreased since the servers were replaced before the server failure occurs due to the wear. The server failure detection rate represents the rate of the server that MEP is configured to the corresponding port and the system has detected the failure quickly by Ethernet OAM, of all the servers that failed through the simulation period. The MEP number was set to 16 , and the server replacement rate was $10 \%$ per week to $50 \%$ per week every $10 \%$. The average value of the server failure locating time was calculated by executing the simulation 100 times for each of the five server replacement rates.

The results of average server failure locating time were 754 seconds for ping only case, 749 seconds for the conventional method case and 732 seconds for the proposed method case, respectively. The change in server replacement rate did not affect the average server failure locating time or the average server failure detection rate. The proposed method reduced the average server failure locating time by $2.8 \%$ and $2.2 \%$ compared to the conventional method case and ping only case, respectively. Also, the proposed method improved the server failure detection rate by 5.32 points in average compared to the conventional method. It can be said that the proposed method increases the average server failure detection rate in data center and reduces the average server failure locating time regardless of the server replacement rate.

Table 2. Server failure locating time and server failure detection rate

\begin{tabular}{cc|ccc|cc}
\hline \multirow{2}{*}{ Server change rate } & \multirow{2}{*}{ Failures } & \multicolumn{3}{|c|}{ Server failure locating time } & \multicolumn{2}{c}{ Server failure detection rate } \\
& & (1) Proposed & (2) Conventional & (3) Ping only & (1) Proposed & (2) Conventional \\
\hline 10\%/week & 1357.65 & $732.69 \mathrm{sec}$. & $748.93 \mathrm{sec}$. & $754 \mathrm{sec}$. & $6.79 \%$ & $1.61 \%$ \\
20\%/week & 700.13 & $731.85 \mathrm{sec}$. & $748.89 \mathrm{sec}$. & $754 \mathrm{sec}$. & $7.05 \%$ & $1.63 \%$ \\
30\%/week & 444.97 & $732.57 \mathrm{sec}$. & $749.04 \mathrm{sec}$. & $754 \mathrm{sec}$. & $6.83 \%$ & $1.58 \%$ \\
40\%/week & 307.11 & $732.30 \mathrm{sec}$. & $749.29 \mathrm{sec}$. & $754 \mathrm{sec}$. & $6.91 \%$ & $1.50 \%$ \\
$50 \% /$ week & 213.47 & $731.99 \mathrm{sec}$. & $748.75 \mathrm{sec}$. & $754 \mathrm{sec}$. & $7.01 \%$ & $1.67 \%$ \\
\hline
\end{tabular}

Also, in order to investigate the effect on the average server failure locating time by the number of MEPs, under the server replacement rate $20 \%$ per week, in the case of the number of MEPs of 8, 16, 32, 64 and 128, each of the simulation is performed 100 times, In each case, the average server failure locating time was measured. The results of this measurement are shown in Figure 3. As a result of this measurement, the average of about 700 server failures occurred in this evaluation. When the number of MEP is increased from 16 to 128 for these server failures, the average server failure locating time was shortened from 749 seconds to 713 seconds when the server was monitored by the Ethernet OAM in the conventional method. On the other hand, when monitoring the server by Ethernet OAM in the proposed method, the average server failure locating time was shortened from 732 seconds to 644 seconds In addition, the average server failure detection rate is improved from $1.6 \%$ to $13 \%$ in the case of the conventional method. Also, it is improved from $6.9 \%$ to $35 \%$ in the case of the proposed method. In the case of the proposed method with 128 MEPs, the Ethernet OAM can identify rapidly the failure factor for about one-third of all server failures. 


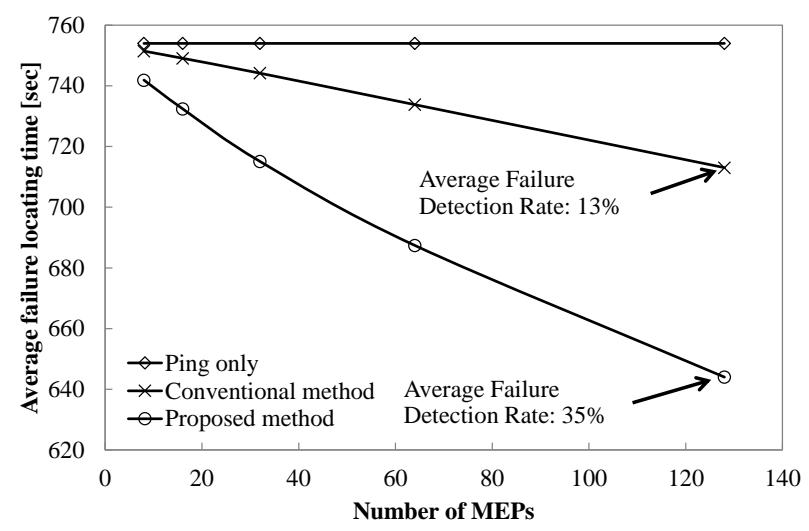

Figure 3. Server Failure Locating Time for the Numbers of Configured MEP

\subsection{Sensitivity Analysis}

The effect on the server failure detection rate is shown in the left part of Figure 4 when the parameter $W_{\text {init }}$ which indicates the magnitude of the initial failure and the amplitude of the change failure used to estimate the server failure rate is different from the actual value. In addition, the right part of Figure 4 shows the effect of the parameter $\sigma_{\text {init }}$ which represents the deviation in the time axis of the initial failure and the change failure used to estimate the server failure rate is different from the actual value. Even if the estimated value of the variance $\sigma_{\text {init }}$ or amplitude $W_{\text {init }}$ is shifted from the actual value, there is no significant change in the evaluation result of the server failure detection rate even in the case of the conventional method and the proposed method.

The result of the sensitivity analysis is described similarly under the environment where the occurrence of the wear-out failure is low, and the initial failure and the change failure are dominant. The simulation parameters which affects the server failure rate is shown in Table 3. The simulation parameters were set so that server failure rate due to the initial server deployment or the change of the server's connection can exceed the server failure rate caused by wear-out failure. The effect of the parameter $W_{\text {init }}$ on the magnitude of the initial failure and the amplitude of the change failure is different from the actual value used to estimate the server failure rate is shown in the left part of Figure 5. In addition, the effect of the parameter $\sigma_{\text {init }}$ on the size of the variance of the time axis of the initial failure and the change failure used to estimate the server failure rate is different from the actual value is shown in the right part of Figure 5. In the case of the proposed method, the server failure detection rate has changed significantly when the deviation $\sigma_{\text {init }}$ is shifted. Specifically, when the ratio of the estimated value of $\sigma_{\text {init }}$ to the actual deviation $\sigma_{\text {init }}$ is 0.5 or less, the server failure detection rate is almost unchanged from the conventional method. On the other hand, when the ratio of deviation $\sigma_{\text {init }}$ is 1 or more, the proposed method exhibits a higher server failure detection rate of about 2.5 points compared to the conventional method.
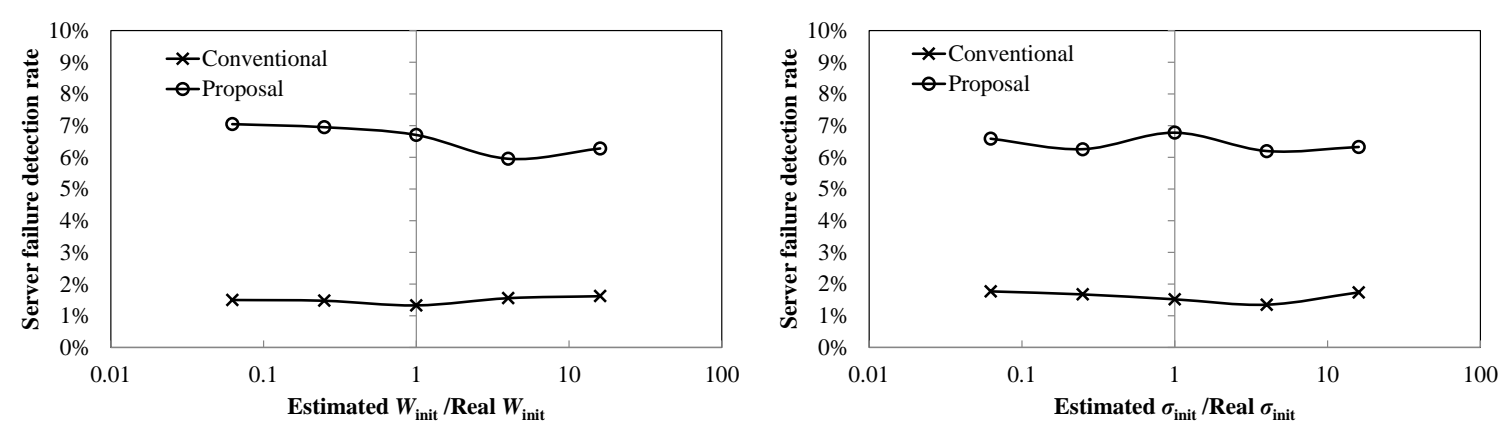

Figure 4. Sensitivity Analysis for $W_{\text {init }}$ (Left) and for $\sigma_{\text {init }}$ (Right) 
Table 3. Simulation parameters in the case where initial failures and change failures are dominant

\begin{tabular}{ll}
\hline Parameter & Value \\
\hline Wear-out failure gradient $a$ & $2 / 3 \mathrm{e}-10$ \\
Initial failure rate amplitude $W_{\text {init }}$ & 0.05 \\
Initial failure rate standard deviation $\sigma_{\text {init }}$ & $5.0 \mathrm{e} 5$ \\
Change failure rate amplitude $W_{\text {chg }}$ & 0.05 \\
Change failure rate standard deviation $\sigma_{\text {chg }}$ & $5.0 \mathrm{e} 5$ \\
\hline
\end{tabular}
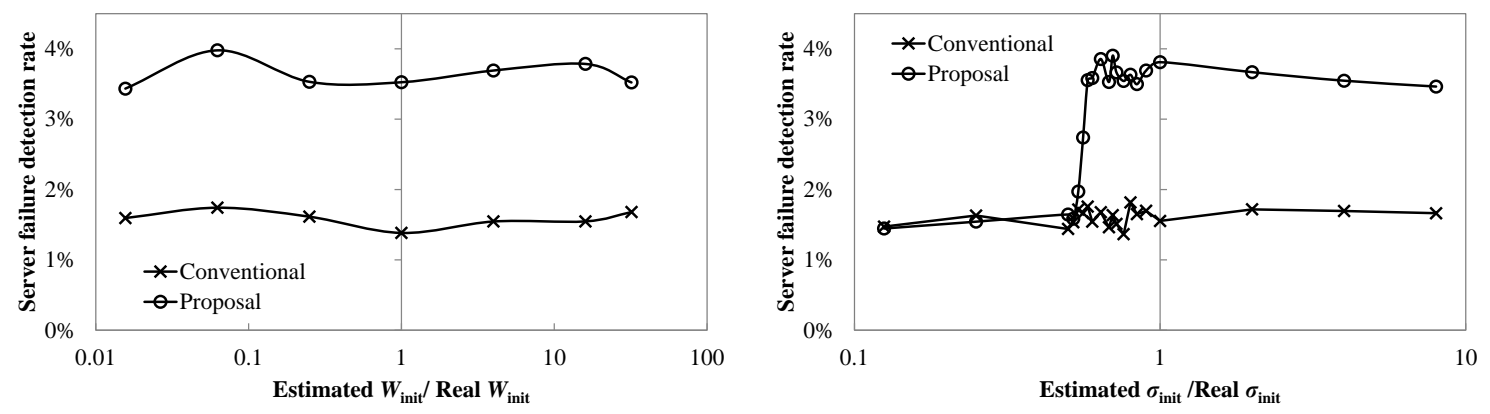

Figure 5. Sensitivity Analysis for $W_{\text {init }}$ (Left) and for $\sigma_{\text {init }}$ (Right) in Less Wear-Out Failure Case

\section{DISCUSSION}

As shown in Figure 3, the average server failure detection rate can be improved from about $7 \%$ to $35 \%$ when the number of MEPs activated in the network of data centers running 1000 servers is increased from 16 to 128 . Therefore, the number of MEPs should be determined according to the target of the failure detection rate or the failure locating time of the data center. Also, we consider the results of the above sensitivity analysis for the case when the initial failure and change failure is dominant. If the ratio of the deviation $\sigma_{\text {init }}$ of the estimated server failure rate to that of the actual server failure rate is less than 0.6, the server failure detection rate of the proposed method decreases to the same level with that of the conventional method. The cause of this behavior can be considered as follows. It is considered that if the actual value of the variance is greater than the estimate, it is more likely that a server that is not set as a monitored target by a MEP will fail. As a result, it is thought that it is the same as the case where the monitored targets by MEPs are selected substantially randomly. To avoid this problem, it is necessary to accurately calculate the deviation $\sigma_{\text {init }}$ of the actual server failure rate in advance based on the failure history of servers in a data center in the past as much as possible.

\section{CONCLUSION}

We proposed a new network monitoring method which coordinates server management and network management. Specifically, we developed a lifecycle-based dynamic virtual network monitoring system that dynamically changes the server to be monitored with Ethernet OAM according to the server failure rate estimated based on the uptime and configuration changes of the server. As an evaluation result by the network simulator, the server failure locating time was reduced by $2.2 \%$ compared to the conventional method of setting fixed monitored targets. It can be said that coordinating server management and network management makes efficient the failure management in the large-scale data center. 


\section{REFERENCES}

Birke, R. et al, 2014. Failure analysis of virtual and physical machines: Patterns, causes and characteristics. Proceedings of 2014 Annual IEEE/IFIP International Conference on Dependable Systems and Networks (DSN'14), Atlanta, U.S.A.

Dini, P. et al, 2004. IP/MPLS OAM: Challenges and directions. Proceedings of IEEE International Workshop on IP Operations and Management, Beijing, China.

Gomes, R.L. et al, 2016. Software defined management of edge as a service networks. In IEEE Transactions on Network and Service Management, vol.13, no.2, pp.226-239.

IEEE 802.1 WG, 2007. IEEE Std 802.1ag-2007 virtual bridged local area networks, amendment 5: Connectivity fault management. IEEE Standards, IEEE Std 802.1ag-2007.

ITU-T, 2015. Operation, administration and maintenance (OAM) functions and mechanisms for Ethernet-based networks. ITU-T Recommendation, ITU-T G.8013/Y.1731.

Katzela, I. and Schwartz, M., 2015. Schemes for fault identification in communication networks. In IEEE/ACM Transactions on Networking, vol.3, no.6, pp.753-764.

Lakhina, A. et al, 2005. Mining anomalies using traffic feature distributions. Proceedings of ACM SIGCOMM 2005, Philadelphia, U.S.A.

Liu, D. et al, 2013. Network traffic anomaly detection using clustering techniques and performance comparison. Proceedings of IEEE CCECE 2013, Regina, Canada.

Luizelli, M.C. et al, 2015. Piecing together the nfv provisioning puzzle: Efficient placement and chaining of virtual network functions. Proceedings of 14th IFIP/IEEE International Symposium on Integrated Network Management (IM 2015), Ottawa, Canada, pp.98-106.

McFarland, M. et al, 2005. Ethernet oam: Key enabler for carrier class metro ethernet services. In IEEE Communications Magazine, vol.43, no.11, pp.152-157.

Mi, H. et al, 2013. Toward finegrained, unsupervised, scalable performance diagnosis for production cloud computing systems. In IEEE Transactions on Parallel and Distributed Systems, vol.24, no.6, pp.1245-1255.

Oppenheimer, D. et al, 2003. Why do internet services fail, and what can be done about it?. Proceedings of 4th USNIX Symposium on Internet Technologies and Systems (USITS'03), Seattle, U.S.A.

Postel, J., 1981. Internet control message protocol (ICMP). IETF RFC, RFC 792.

Schroeder, B. and Gibson, G.A., 2006. A large-scale study of failures in high-performance computing systems. Proceedings of 2006 International Conference on Dependable Systems and Networks (DSN'06), Philadelphia, U.S.A.

Schroeder, B. and Gibson, G.A., 2007. Disk failures in the real world: What does an MTTF of 1,000,000 hours mean to you?. Proceedings of FAST'07, San Jose, U.S.A.

Tang, Y. et al, 2005. Active integrated fault localization in communication networks. Proceedings of 9th IFIP/IEEE Interna tional Symposium on Integrated Network Management (IM 2005), Nice, France, pp.543-556.

Thottan, M. and Ji, C., 2003. Anomaly detection in IP networks. In IEEE Transactions on Signal Processing, vol.51, no.8, pp.2191-2204.

Varga, P. and Moldovan, I., 2007. Integration of service-level monitoring with fault management for end-to-end multi-provider ethernet services. In IEEE Transactions on Network and Service Management, vol.4, no.1, pp.28-38.

Yang, J. and Sun, F.B., 1999. A comprehensive review of hard-disk drive reliability. Proceedings of Annual Reliability and Maintenability Symposium (RAMS), Washington, DC, U.S.A., pp.403-409. 